\title{
Grado de Agudeza Visual de los estudiantes de la Carrera de Enfermería de la Universidad Católica de Cuenca extensión Cañar
}

\section{Grade of Visual sharpness of the students of the Career of Infimary of the Catholic University of Cuenca extension Cañar}

\author{
Rojas Verdugo, Paola Erica* y Martínez Santander, Carlos José \\ Universidad Católica de Cuenca \\ Cañar, 030201, Ecuador \\ *eprojasv@ucacue.edu.ec
}

\begin{abstract}
Resumen
Los problemas visuales pueden traer muchas complicaciones en la vida diaria de las personas y más aún en una población de estudiantes. Puede causar hasta fracaso académico si no se da la importancia con la que se debe tratar este problema.La presente investigación da a conocer el grado de agudeza visual de los estudiantes de la carrera de Enfermería de la Universidad Católica de Cuenca extensión Cañar. El objetivo es determinar el grado de visualización que tienen los estudiantes y plantearles alternativas de mejoramiento como solución a este problema Se realizó una investigación exploratoria, la cual se ejecutó en 3 etapas:1) utilizar la adecuada metodología para llegar a la comunidad estudiantil. 2) Recopilación, análisis, tabulación e interpretación de información. 3) Elaboración de la propuesta para obtener una guía de alternativas para mejorar el problema.El análisis de los resultados demuestra que la población estudiantil de la Universidad, se encuentra en un rango mayor al $53 \%$ con problemas de agudeza visual, por lo que, se desarrolló como propuesta talleres, charlas, eventos que promuevan el conocimiento sobre la agudeza visual, así también el manejo de una guía sobre este tema que mediante texto y gráficos permita que los estudiantes, padres de familia, y comunidad en general entiendan mejor el tema y consecuencias que pueden ocasionar. Se concluye que dentro de la comunidad educativa existen problemas de impedimento visual leve que no son tomadas en cuenta por los mismos llegando a generar un problema más agudo a futuro.
\end{abstract}

Palabras clave: Agudeza, Visual, Metodología, Agudeza Visual Estereoscópica [Ave], Oftalmológica.

\begin{abstract}
Visual problems can bring many complications in the daily life of people and even more so in a population of students. It can even cause academic failure if you do not give the importance with which this problem should be addressed. The present investigation reveals the degree of visual acuity of the students of the Nursing career of the Catholic University of Cuenca Cañar extension. The objective is to determine the degree of visualization that the students have and to propose them alternatives of improvement as a solution to this problem. An exploratory research was carried out, which was carried out in 3 stages: 1) to use the appropriate methodology to reach the student community. 2) Compilation, analysis, tabulation and interpretation of information. 3) Elaboration of the proposal to obtain a guide to alternatives to improve the problem. The analysis of the results shows that the student population of the University, is in a range greater than $53 \%$ with problems of visual acuity, reason why, it was developed like proposal workshops, talks, events that promote the knowledge on the visual acuity, as well as the management of a guide on this topic that, through text and graphics, allows students, parents, and the community in general to better understand the issue and its consequences. It is concluded that within the educational community there are problems of mild visual impairment that are not taken into account by them, generating a more acute problem in the future.
\end{abstract}

Key words: Acute, Visual, Methodology, Stereoscopic Visual Actuation [Ave], Ophthalmology.

\section{Introducción}

Los servicios de urgencias oftalmológicas en el mundo registran un vertiginoso crecimiento de una población con problemas visuales. Se estima que mucho de estos problemas pudieron ser evitados si se trataban a tiempo.
Estos problemas han conllevado a una ceguera en jóvenes, desgraciadamente los factores asociados a estos problemas en investigaciones realizadas son: problemas económicos y sociales difíciles de cuantificar. ${ }^{1}$ 
Se ha demostrado que muchos de los fracasos escolares o académicos han sido producidos por problemas oculares, agudeza visual que no se ha tratado a tiempo. El déficit de la visión tiene un alto impacto en todos los aspectos de la vida, sobre todo en el aprendizaje. Por esta razón es de importancia que el personal de salud en atención primaria en salud y en nuestro caso personal de enfermería, participen en la generación de programas de detección temprana de problemas de agudeza visual. ${ }^{2}$

Con estos contenidos es importante manifestar que se presente el siguiente trabajo denominado: Grado de Agudeza Visual de los estudiantes de la Carrera de Enfermería de la Universidad Católica de Cuenca Extensión Cañar periodo Diciembre-2015, Marzo-2016, que tienen como objetivo determinar cuántos y cuál es el grado de visualización que tienen los estudiantes y platearles alternativas de mejoramiento y solución a este problema. Es por ello que en función de los resultados obtenidos se plantearán y analizarán las estrategias, resultados y se determinará el impacto de estos resultados en el accionar en los estudiantes de la carrera de enfermería, a través de indicadores de resultado, mismos que pretenden establecer la factibilidad y la aplicación de métodos para solucionar problemas de agudeza visual.

La necesidad de una proyección epidemiológica que permita establecer una primera aproximación de la frecuencia de estos defectos de refracción, es decir la evaluación del estado clínico epidemiológico en los estudiantes universitarios es de mucha utilidad; por lo tanto proveer a los estudiantes universitarios el diagnóstico y resolución de sus problemas visuales a temprana edad y en forma oportuna, será una mejora en las oportunidades de vida.

Hoy se sabe que sobre el $85 \%$ del aprendizaje de una estudiante se da a través del sentido de la vista, por lo tanto mantener una buena salud visual desde los primeros años de escolaridad tiene un efecto directo sobre el aprendizaje.

Para valorar los problemas visuales de los estudiantes se realizó el examen de agudeza visual para medir el nivel de visión que poseen utilizando el optotipo de Snellen a 6 metros de distancia, con lo cual encontramos varios problemas visuales dentro de la población universitaria.

\section{Método}

Es un estudio cuantitativo, observación descriptiva que da a conocer el contexto de forma general de la situación actual a más de que se está en contacto directo con el objeto de estudio. De acuerdo al fin que persigue la investigación es básica ya que está orientada a lograr un nuevo conocimiento de manera sistemática y metódica, con el único objetivo de ampliar el conocimiento.

Esta investigación se sustentará en la investigación explicativa, descriptiva y explicativa debido a que se debe profundizar, conocer directamente el objeto a estudio y conocer la relación causa-efecto, además fundamenta con la investigación documental a través de técnicas como encuestas entrevistas y fichas, con la investigación de campo que se recolecto la información de los estudiantes de la UCACUE.

Tomando como muestra a los estudiantes de la escuela de enfermería de la Universidad católica de Cuenca extensión Cañar con un número de 270 estudiantes realizando el cálculo matemático del muestreo probabilístico que me arrojo un numero de 45 alumnos que se les efectuó la encuesta para tener resultados confiables.

\section{Resultados}

De acuerdo a las encuestas realizadas a los estudiantes se obtienen los siguientes resultados para lo cual hemos visto necesario analizar las respuestas con mayor y menor porcentajes de las mismas que nos indicaran acerca de lo que saben o desconocen los encuestados acerca de la misma:

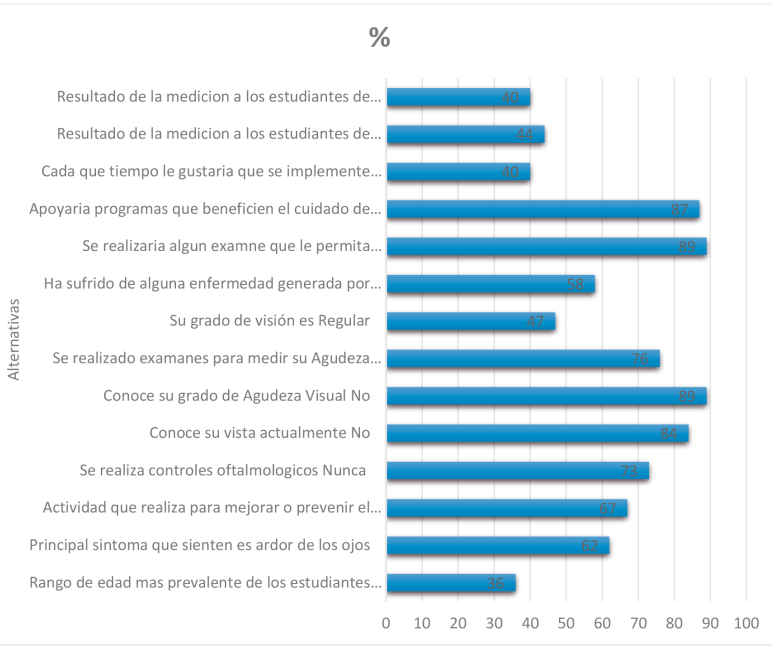

Fig. 1.

Con el gráfico 1 podemos observar que los estudiantes padecen de enfermedades visuales que pueden estar empeorando su aprendizaje, indicando que les gustaría una revisión oftalmológica cada seis meses pero que no estarían dispuestos apoyar a una institución benéfica que les ayude con la revisión, a pesar de esto les gustaría que les realicen exámenes debido a que por esta dificultad visual sufren síntomas que les está afectando en su vida diaria, a más de ello suelen manifestar que nunca se han hecho controles visuales y ni siquiera conocen acerca de su grado de agudeza visual; y que no realiza ninguna actividad para mejorar esta visión indicando que son estudiantes que tienen una edad entre 21 a 23 años siendo estos los más jóvenes y quienes no dan importancia al cuidado de su visión. 


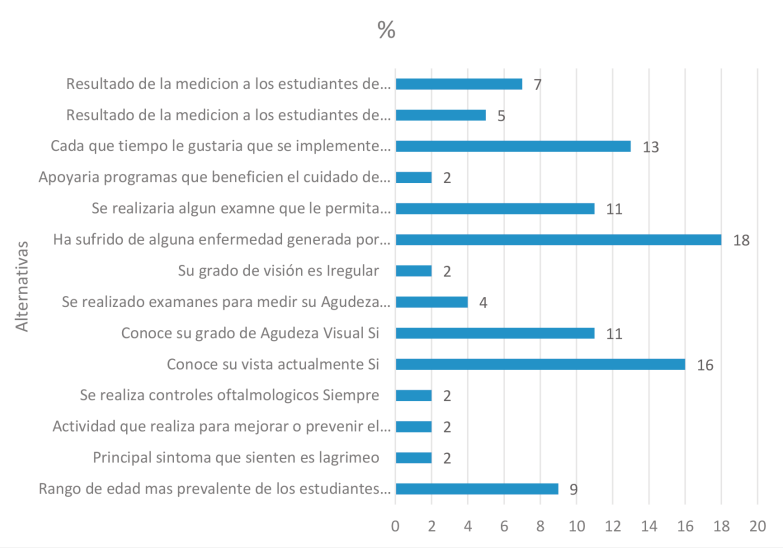

Fig. 2.

Por lo contrario al grafico 2 podemos manifestar que los estudiantes padecen de una mala visión tanto lejana como cercana solicitando así que se realice una revisión oftálmica cada mes, ya que nunca se han realizado exámenes visuales y manifiestan no haber sufrido ninguna alteración o síntoma que altere su visión o que provoque una alteración a pesar de que su visión es irregular algunos estudiantes indican que están al tanto de su grado agudeza visual; podemos recalcar que a pesar de ser la mayoría estudiantes de un rango de edad de 24 a 26 años ellos están dispuestos a apoyar programas que beneficien su salud visual.

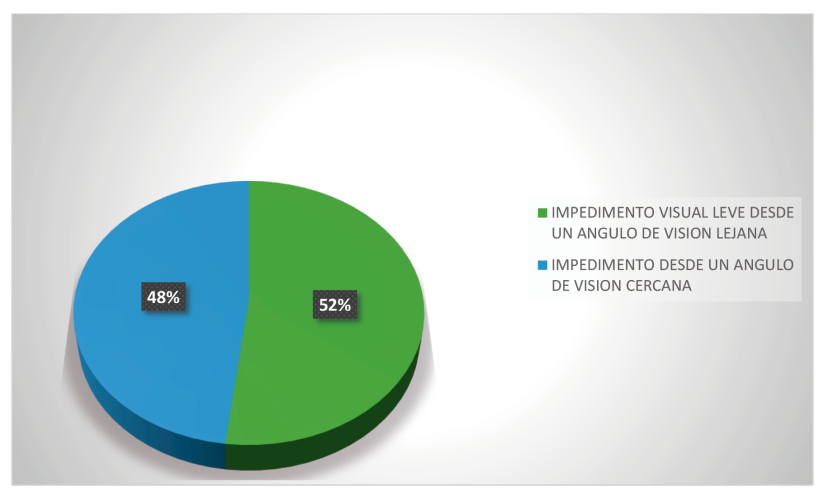

Fig. 3.

Como se aprecia en el gráfico 3 podemos observar que la mayor parte de estudiantes encuestados padecen de problemas con un $52 \%$ de impedimento visual leve desde un ángulo de visión lejana y es debido a esto que los estudiantes presentan problemas de aprendizaje o no prestan atención a la misma ya que hacen un esfuerzo grande con la visión para poder enfocarse en lo que el docente escribe en el pizarrón.

Y también podemos observar que el $48 \%$ padece de un impedimento de un ángulo de visión cercana y como consecuencia no existe un buen aprendizaje por parte de los alumnos; es por ello que en la mayoría de los casos los estudiantes necesitan utilizar lentes para mejorar la visión de los mismos y mejorar el aprendizaje.

\section{Análisis de Confiabilidad y Validez}

Una vez desarrollado este trabajo de investigación, con los datos recolectados y tabulados, graficados, analizados y comprendidos, los cuales se encuentran debidamente representados de forma estadística y porcentual en las gráficas insertadas.

Se determina también que la hipótesis planteada verifica que los estudiantes están conscientes de que podemos sufrir este trastorno visual, por lo que, estar preparados y dispuestos a mejorar nuestra condiciones de vida para que ello repercuta en la salud integral del ser humano y no tan solo en la de nuestros órganos de la vista siendo la hipótesis general

\section{Afirmativa}

En cuanto la población estudiantil de la Universidad, la cual se ve muy interesada en buscar soluciones, para que el rendimiento académico de los estudiantes afectados por problemas de agudeza visual mejore, todos están en un $93 \%$ dispuestos a participar en eventos que promuevan el conocimiento sobre la agudeza visual, así también en el manejo de una guía sobre este tema que mediante texto y gráficos permita que los estudiantes entiendan mejor sobre el tema y tengan un materia en dónde consultar sobre estos problemas, teniendo como hipótesis particular AFIRMATIVA.

\section{Conclusiones}

EEn base a los resultados obtenidos se determinó que en un $53 \%$ de los estudiantes de la carrera de enfermería tienen un impedimento visual leve, desde un ángulo de visión lejana, influyendo que los estudiantes tienen estos problemas pero no se interesa por conocer el estado de su vista, de esta forma el problema va creciendo afectando la salud visual del estudiante y por consiguiente su desarrollo dentro del ambiente educativo.

En cuanto a los resultados sobre un ángulo de visión cercana el $49 \%$ de los estudiantes presentan estos problemas, que es considerado como un impedimento visual disminuido, por consiguiente se interpreta la necesidad de fomentar el tratamiento y cuidado visual mediante vistas al oftalmólogo, así como los exámenes visual pertinentes, así como el fomento de una unidad médica para el cuidado y protección de la vista que acuda al centro universitario.

Se pudo entregar una guía educativa sobre la aguza visual, este tipo de material permite que los estudiantes tengan un conocimiento más amplio sobre este problema, puedan tratarlo, prevenirlo así como que este documento sirva para difundir para todos los demás compañeros y sociedad en general para que conozcan sobre los problemas generados por la agudeza visual y más aún en el ámbito educativo como estudiante. 


\section{Referencias Bibliográficas}

1. y Maria González Mesa y Elvira Machado Héctor y María Llull Tombo MCG. Pronóstico visual según clasificación estandarizada en pacientes ingresados por traumatismos oculares. Medisur. 2009;7(6).

2. Rincón IA, Rodríguez NC. Tamización de salud visual en población infantil: prevención de la ambliopía;18(4):210 217.

3. Acevedo Figueroa L. ri.uaq.mx/bitstream;

4. Adformacion. CUESTIONARIO DE MASLACH.;.

5. Arroyo G. Soluciones para la baja visión;.

6. ASHA. American Sexual Health Association;

7. Attewell A. Florence Nightingale; Temperamentvm;

8. Ballesteros C. La Sexualidad;.

9. Banda O. El significado de la sexualidad en adolescentes;

10. Bermúdez M, Castro A, Madrid J. Análisis de la conducta sexual de adolescentes autóctonos e inmigrantes latinoamericanos en España;

11. Berreceli M. Parasitología;.

12. Bibliotecraio Rd. Síndrome del Burnout;.

13. Bohigian G. Antecedentes Historicos de la Agudeza Visual;.

14. Borja T. "Sexualidad: diversidad y procesos de desarro1lo". CECAFEC (Centro Ecuatorianode Capacitación y Formacion de Educadores de la Calle);.

15. Cardo U. Influencia del estrés y el síndrome de Burnout en la calidad de los servicios de enfermería. CEEIS;

16. Medicina Sy. EL ESTRÉS CAUSAS Y CONSECUENCIAS Y TRATAMIENTO STRESS SINTOMAS;.

Recibido: 31 de mayo de 2017

Aceptado: 18 de julio de 2017 